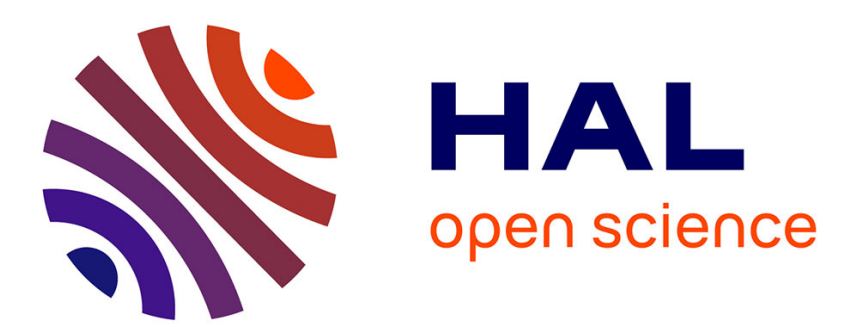

\title{
Penetration of the electric and magnetic field components of Schumann resonances into the ionosphere
}

\author{
V. Grimalsky, S. Koshevaya, A. Kotsarenko, R. Perez Enriquez
}

\section{To cite this version:}

V. Grimalsky, S. Koshevaya, A. Kotsarenko, R. Perez Enriquez. Penetration of the electric and magnetic field components of Schumann resonances into the ionosphere. Annales Geophysicae, 2005, 23 (7), pp.2559-2564. hal-00317898

\section{HAL Id: hal-00317898 \\ https://hal.science/hal-00317898}

Submitted on 14 Oct 2005

HAL is a multi-disciplinary open access archive for the deposit and dissemination of scientific research documents, whether they are published or not. The documents may come from teaching and research institutions in France or abroad, or from public or private research centers.
L'archive ouverte pluridisciplinaire HAL, est destinée au dépôt et à la diffusion de documents scientifiques de niveau recherche, publiés ou non, émanant des établissements d'enseignement et de recherche français ou étrangers, des laboratoires publics ou privés. 


\title{
Penetration of the electric and magnetic field components of Schumann resonances into the ionosphere
}

\author{
V. Grimalsky ${ }^{1}$, S. Koshevaya ${ }^{2}$, A. Kotsarenko ${ }^{3}$, and R. Perez Enriquez ${ }^{3}$ \\ ${ }^{1}$ National Institute for Astrophysics, Optics, and Electronics, Puebla 72000, Pue., Mexico \\ ${ }^{2}$ Autonomous University of Morelos, CIICAp, Av.Universidad 1001, Cuernavaca 62210, Mor., Mexico \\ ${ }^{3}$ UNAM, Center of Geoscience, Juriquilla, P.O. 1-742, Queretaro 76230, Qro, Mexico
}

Received: 16 September 2004 - Revised: 21 July 2005 - Accepted: 17 August 2005 - Published: 14 October 2005

\begin{abstract}
A penetration of electric and magnetic fields of the first global electromagnetic ELF resonance into the ionosphere in the cavity Earth-ionosphere is investigated numerically. It is shown that a penetration height for magnetic components is $2-3$ times greater than for electric components and it depends essentially on the value of the geomagnetic field and its orientation with respect to the normal to the Earth's surface. A penetration height for the electric field is about 50 $\div 70 \mathrm{~km}$, and for the magnetic field it is $120 \div 240 \mathrm{~km}$. An influence of variations of the conductivity of the ionosphere at the daytime and nighttime and under different solar activity on a penetration of the fields of the first Schumann resonance has been investigated.
\end{abstract}

Keywords. Electromagnetics (Guided waves) - Ionosphere (Ionosphere-atmosphere interactions; Wave propagation)

\section{Introduction}

Schumann (global) electromagnetic (EM) resonances in the cavity Earth - ionosphere play an important role in the lithosphere - atmosphere - ionosphere - magnetosphere coupling (Hayakawa, 1999; Bliokh et al., 1980; Nickolaenko and Hayakawa, 2002; Nickolaenko, 1997). A very rough approximation to describe the properties of the Schumann resonances is an assumption of the ideal conductivity of both the Earth's surface and of the ionosphere E-layer. Such an approximation gives a possibility to estimate the values of the resonant frequencies $(8 \mathrm{~Hz}, 14 \mathrm{~Hz}, 20 \mathrm{~Hz}$, and $26 \mathrm{~Hz})$. Some specifications were put forward by means of the resonator perturbation theory (Bliokh et al., 1980; Nickolaenko and Hayakawa, 2002; Nickolaenko, 1997; Vainshtein, 1988; Sentman, 1995), where a formalism of the tensor surface impedance of the ionosphere plasma was applied. Such a theory gives a possibility to estimate the quality factor of

Correspondence to: V. Grimalsky

(vgrim@inaoep.mx) the resonances (about 5) and to investigate an excitation of the Schumann resonator by external current sources (atmospheric electricity). An excitation of Schumann resonances due to thunderstorm activity has been investigated in the papers (Füllekrug and Constable, 2000; Füllekrug and Reising, 1998; Mushtak and Williams, 2002). Also, there are some papers devoted to a penetration of electric and magnetic fields of the Schumann resonances into the lower ionosphere, where an exponential (or more complicated) approximation of the isotropic conductivity (and, therefore, effective permittivity) of the ionosphere plasma was used (Nickolaenko and Hayakawa, 2002; Sentman, 1995). When neglecting an influence of the geomagnetic field, a penetration height for the electric field was estimated as $50 \div 70 \mathrm{~km}$, and for the magnetic field it was about $80 \div 100 \mathrm{~km}$. The modification of the properties of resonance modes due to solar activity has been observed and investigated within an approximation of the isotropic conductivity of the ionosphere (Roldugin, 2003). Also, the dissipation of Schumann modes has been estimated from the approximation of isotropic ionosphere (Sentman, 1983). Note that an essential influence of the geomagnetic field on a penetration of extremely low frequency (ELF) electromagnetic fields into the ionosphere was mentioned in the early survey (Madden and Thompson, 1965). Moreover, some authors, who used an isotropic approximation, mentioned an importance of the anisotropy of the conductivity of the ionosphere (Sentman, 1983; Mushtak and Williams, 2002).

The vertical profile of the conductivity of the ionosphere is not ideally sharp; its characteristic scale of variation is about $10 \div 30 \mathrm{~km}$. A strong anisotropy of conductivity of the ionosphere, due to the presence of the geomagnetic field, becomes essential at the heights of $70 \div 80 \mathrm{~km}$ and more (Madden and Thompson, 1965; Rich and Basu, 1985; Al'pert, 1972). Thus, a modification of the vertical profile of the anisotropic conductivity of the lower ionosphere, due to various processes, may lead to a change in properties of the global resonance modes. It is clear that electric and 


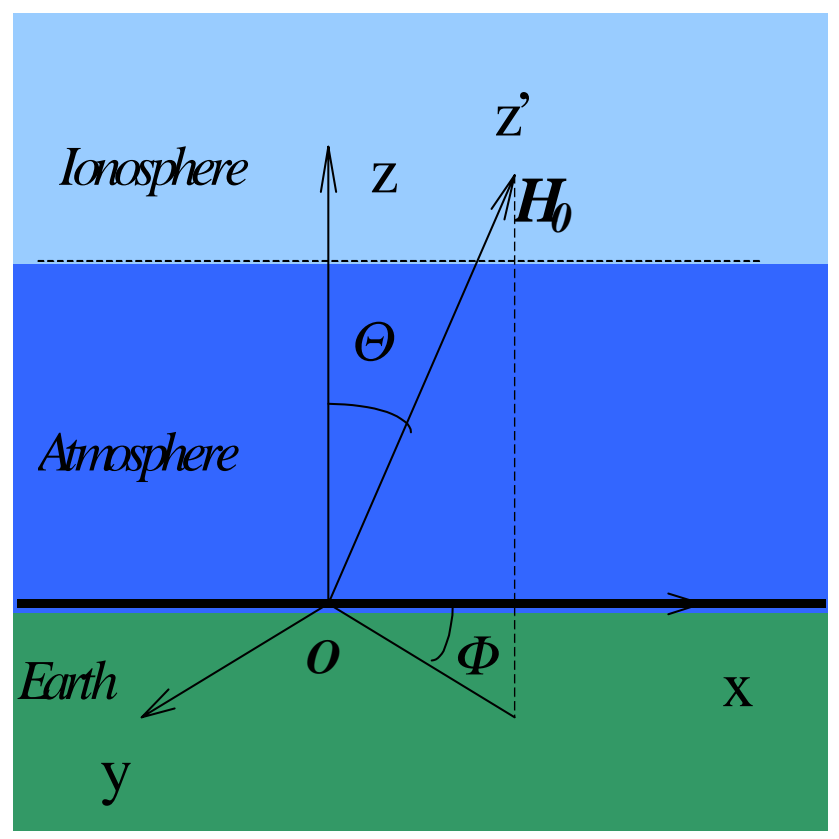

Fig. 1. Geometry of the problem. (XYZ) are local coordinates.

magnetic components of the Schumann modes possess different vertical distributions. But it is important to know more exactly about the difference between the corresponding penetration heights in the presence of the geomagnetic field.

In this paper, the vertical profiles of the electric and magnetic fields of the first global Schumann resonance in the cavity Earth - ionosphere are investigated numerically in the cases of various vertical profiles of the electron concentration of the ionosphere. The anisotropy of conductivity due to the presence of the geomagnetic field is taken into account. It is shown that the horizontal magnetic components of the resonance mode can penetrate highly into the E-layer of the ionosphere and even into the F-layer. The penetration height depends on the value and orientation of the local geomagnetic field; it is about $50 \div 70 \mathrm{~km}$ for electric components and $120 \div 240 \mathrm{~km}$ for magnetic components. For that reason, variations of the conductivity of the ionosphere D-layer may lead to essential changes in vertical profiles of both electric and magnetic components of the modes. Correspondingly, variations in the conductivity of the ionosphere E-layer may lead to changes in the vertical profiles of the magnetic components.

\section{Basic equations}

The global resonances in the cavity Earth - ionosphere possess the resonant frequencies in the ELF range. The lowest (fundamental) frequency is about $8 \mathrm{~Hz}$ (Bliokh et al., 1980; Nickolaenko and Hayakawa, 2002; Nickolaenko, 1997). Due to a global character, a distribution along the Earth's surface is smooth, and a characteristic scale is the Earth's radius $R_{E}=6400 \mathrm{~km}$. The vertical scale of the field distribu- tion depends on the profile of the ionosphere and it is about $10 \div 30 \mathrm{~km}$. Thus, these scales differ from each other very essentially. This gives a possibility to consider the problem of the penetration of the EM components of the resonance mode into the ionosphere, approximately within the assumptions that the Earth's surface is locally plane and a horizontal dependence of EM components is the same with a change in the vertical coordinate.

Let us estimate an applicability of the plane approximation of the Earth's surface. A deviation of the tangential line from the curved Earth's surface is $z_{s}=L_{x}^{2} /\left(2 R_{E}\right)$ at the horizontal distance $L_{x}$. The parameter $L_{x}$ describes the horizontal scale where the ionosphere can be assumed as vertically nonuniform only. Let us choose the value $L_{x}=400 \mathrm{~km}$, which is twice as large as the possible height of a penetration of magnetic fields of the global resonances into the ionosphere. Here, $z_{s} \approx 12 \mathrm{~km}$. Therefore, $z_{s}$ is smaller than a characteristic vertical scale of the ionosphere $(20 \div 30 \mathrm{~km})$, and a curvature of the Earth's surface may be considered as a perturbation. A dependence of the distributions of horizontal components of the magnetic field of the global Schumann resonances on the local height of the ionosphere D-layer was confirmed experimentally (Sentman and Fraser, 1991).

Let us denote the local vertical axis as $O Z$, horizontal axes are $O X$ and $O Y$ (see Fig. 1), and assume that the horizontal axes are chosen so that the local dependence of the mode components in the horizontal plane is $\sim \exp \left(\mathrm{ik}_{x} x\right)$. A temporal dependence is $\sim \exp (\mathrm{i} \omega \mathrm{t})$, where $\omega$ is an angular resonant frequency, which is generally complex, due to the damping of the resonant mode. But, to consider the vertical profile of the mode, it is necessary to use only the real part of the resonant frequency in the expressions of the tensor of dielectric permittivity (Vainshtein, 1988). Note that simulations with the resonant frequency including the image part (within 20\% of the real part) do not change the results essentially.

The components of a dielectric tensor in the coordinate frame connected with the direction of the geomagnetic field take a form:

$$
\begin{aligned}
& \hat{\varepsilon}^{\prime}(\omega, z)=\left(\begin{array}{rrl}
\varepsilon_{1} & \varepsilon_{h} & 0 \\
-\varepsilon_{h} & \varepsilon_{1} & 0 \\
0 & 0 & \varepsilon_{3}
\end{array}\right) ; \\
& \varepsilon_{1}=1-\frac{\omega_{p e}^{2}\left(\omega-i v_{e}\right)}{\omega\left(\left(\omega-i v_{e}\right)^{2}-\omega_{H e}^{2}\right)}-\frac{\omega_{p i}^{2}\left(\omega-i v_{i}\right)}{\omega\left(\left(\omega-i v_{i}\right)^{2}-\omega_{H i}^{2}\right)} ; \\
& \varepsilon_{3}=1-\frac{\omega_{p e}^{2}}{\omega\left(\omega-i v_{e}\right)}-\frac{\omega_{p i}^{2}}{\omega\left(\omega-i v_{i}\right)} ; \\
& \varepsilon_{h}=i\left(\frac{\omega_{p e}^{2} \omega_{H e}}{\omega\left(\left(\omega-i v_{e}\right)^{2}-\omega_{H e}^{2}\right)}-\frac{\omega_{p i}^{2} \omega_{H i}}{\omega\left(\left(\omega-i v_{i}\right)^{2}-\omega_{H i}^{2}\right)}\right) ; \\
& \omega_{p e}^{2}=\frac{4 \pi e^{2} n_{0}}{m_{e}}, \omega_{p i}^{2}=\frac{4 \pi e^{2} n_{0}}{m_{i}}, \omega_{H e}=\frac{e H_{0}}{m_{e} c}, \omega_{H i}=\frac{e H_{0}}{m_{i} c} .
\end{aligned}
$$

Here $n_{0}$ is the electron concentration; $v_{e, i}$ are collision frequencies for electrons and ions, respectively; $m_{e, i}$ are their masses; $\omega_{p e, i}$ and $\omega_{H e, i}$ are corresponding plasma and cyclotron frequencies (Nickolaenko and Hayakawa, 2002). 
The set of Maxwell's equations for the electric $\left(E_{x, y, z}\right)$ and magnetic $\left(H_{x, y, z}\right)$ components of the mode takes the form:

$$
\begin{aligned}
& \frac{\partial^{2} E_{x}}{\partial z^{2}}-i k_{x} \frac{\partial E_{z}}{\partial z}+k_{0}^{2} D_{x}=0 ; \\
& \frac{\partial^{2} E_{y}}{\partial z^{2}}-k_{x}^{2} E_{y}+k_{0}^{2} D_{y}=0 ; \\
& i k_{x} \frac{\partial E_{x}}{\partial z}+k_{x}^{2} E_{z}-k_{0}^{2} D_{z}=0 ; \\
& D_{j}=\varepsilon_{j l}(\omega, z) E_{l} ; k_{0}=\omega / c ; \boldsymbol{E} \sim \exp \left(i k_{x} x\right) .
\end{aligned}
$$

Here $\varepsilon_{j l}(\omega, \mathrm{z})$ are the components of the tensor of the dielectric permittivity, $\varepsilon^{\wedge}(\omega, \mathrm{z})$, of the atmosphere and the ionosphere in the coordinate frame $(X Y Z) ; D_{j}$ are the components of the electric induction vector. The components $\varepsilon_{j l}$ depend essentially on the vertical coordinate $z$ and weakly on the horizontal coordinates $x, y$. An absolute system of units is used here, where the units for the electric and magnetic fields are the same. It is possible to express all the components of the mode through $D_{z}$ and $E_{y}$ and to obtain the coupled equations for them:

$$
\begin{aligned}
& \frac{d}{d z}\left(\frac{\varepsilon_{33}}{\Delta} \frac{d D_{z}}{d z}\right)+i k_{x} \frac{d}{d z}\left(\frac{\varepsilon_{13}}{\Delta} D_{z}\right)+i k_{x} \frac{\varepsilon_{31}}{\Delta} \frac{d D_{z}}{d z} \\
& -i k_{x} \frac{d}{d z}\left(e_{1} E_{y}\right)+\left(k_{0}^{2}-k_{x}^{2} \frac{\varepsilon_{11}}{\Delta}\right) D_{z}-k_{x}^{2} e_{3} E_{y}=0 ; \\
& \frac{d^{2} E_{y}}{d z^{2}}-\frac{i k_{0}^{2}}{k_{x}} e_{4} \frac{d D_{z}}{d z}-k_{0}^{2} e_{2} D_{z}+ \\
& +\left(k_{0}^{2} \tilde{\varepsilon}_{22}-k_{x}^{2}\right) E_{y}=0 ; \\
& E_{x}=\frac{i}{k_{x}} \frac{\varepsilon_{33}}{\Delta} \frac{d D_{z}}{d z}-\frac{\varepsilon_{13}}{\Delta} D_{z}+e_{1} E_{y} ; H_{x}=-\frac{i}{k_{0}} \frac{d E_{y}}{d z} ; \\
& E_{z}=-\frac{i}{k_{x}} \frac{\varepsilon_{31}}{\Delta} \frac{d D_{z}}{d z}+\frac{\varepsilon_{11}}{\Delta} D_{z}+e_{3} E_{y} ; \\
& H_{y}=\frac{k_{0}}{k_{x}} D_{z} ; H_{z}=-\frac{k_{x}}{k_{0}} E_{y} ;
\end{aligned}
$$

The following notations are used:

$$
\begin{aligned}
& e_{1}=\frac{1}{\Delta}\left(\varepsilon_{13} \varepsilon_{32}-\varepsilon_{12} \varepsilon_{33}\right) ; e_{2}=\frac{1}{\Delta}\left(\varepsilon_{21} \varepsilon_{13}-\varepsilon_{11} \varepsilon_{23}\right) ; \\
& e_{3}=\frac{1}{\Delta}\left(\varepsilon_{31} \varepsilon_{12}-\varepsilon_{11} \varepsilon_{32}\right) ; e_{4}=\frac{1}{\Delta}\left(\varepsilon_{23} \varepsilon_{31}-\varepsilon_{21} \varepsilon_{33}\right) ; \\
& \Delta=\varepsilon_{11} \varepsilon_{33}-\varepsilon_{13} \varepsilon_{31} \tilde{\varepsilon}_{22}=\varepsilon_{22}+\varepsilon_{21} e_{1}+\varepsilon_{23} e_{3} .
\end{aligned}
$$

The Earth's surface is assumed to be ideally conductive, therefore, the boundary conditions are: $E_{y}(z=0)=0, D_{z}$ $(z=0)=D_{z 0}=1 ; E_{y}\left(z=L_{z m}\right)=0, D_{z}\left(z=L_{z m}\right)=0$ in the highly conductive F-layer of the ionosphere. The results do not depend on the choice of the upper boundary $L_{z m}$, when $L_{z m}>250 \mathrm{~km}$. The problem is to investigate the vertical profiles of all the components of the first Schumann resonance mode.

The value of the resonant frequency of the lowest EM Schumann mode has been chosen as $f \approx 8 \mathrm{~Hz}\left(\omega=50 \mathrm{~s}^{-1}\right)$, known from experimental data (Bliokh et al., 1980; Nickolaenko, 1997). A horizontal distribution of the mode is determined by the parameter $k_{x} \approx R_{E}^{-1}$, and it is approximately equal to $k_{0}=\omega / \mathrm{c}$. Small deviations (up to $30 \%$ ) from the values of $\omega$ and $k_{x}$ above do not change the main qualitative results of the paper.
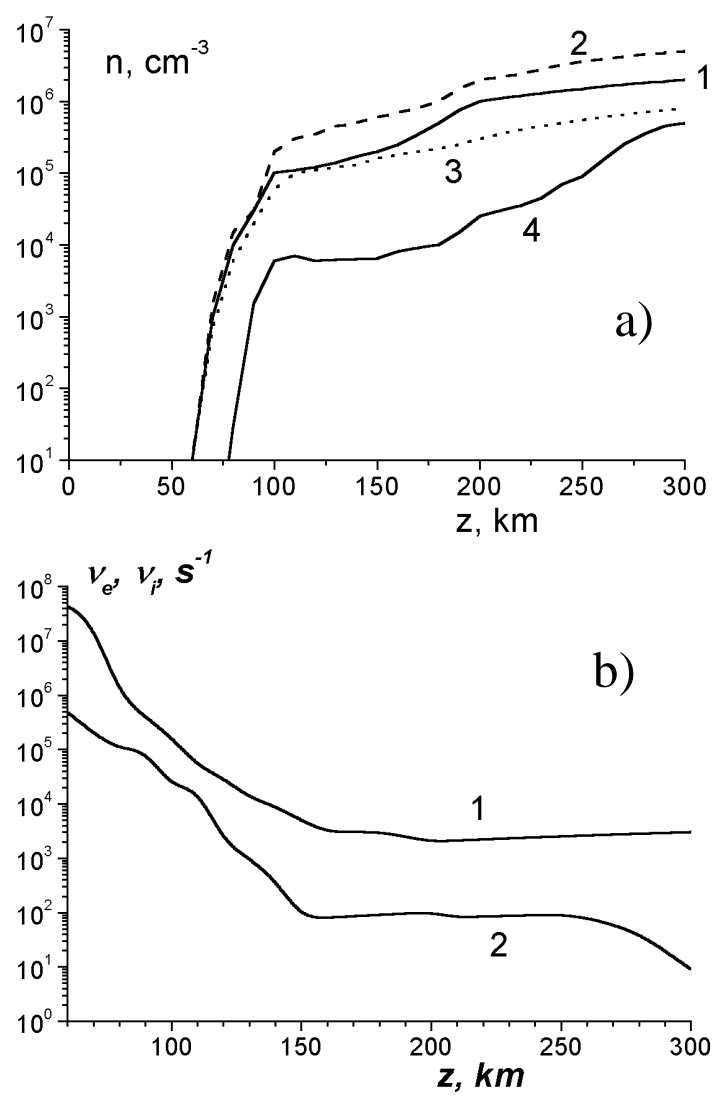

Fig. 2. Part (a). Vertical dependence of the concentration of electrons used in the simulations. Curve 1 is at the daytime (normal solar activity), curve 2 is at the daytime (maximum solar activity), curve 3 is at the daytime (minimal solar activity), curve 4 is at the nighttime. Part (b). Vertical dependencies of electron (curve 1) and ion (curve 2) collision frequencies used in the simulations.

\section{Numerical simulations}

Numerical simulations have been provided by means of a finite difference approximation of Eq. (2). The obtained threediagonal matrix linear equations have been solved by the matrix factorization method. The steps of the calculations are $50 \mathrm{~m}-3 \mathrm{~m}$. The vertical profiles of the concentration of electrons used in the simulations are given in Fig. 2. The data on the concentrations of electrons, collision frequencies, and average molar masses have been taken from Rich and Basu (1985); Al'pert (1972). One can see that there exists an essential difference between the values at the daytime under different solar activity and at the nighttime. The vertical profiles of the absolute values of the components of the complex dielectric permittivity $\left|\varepsilon_{1,3, h}\right|$ in the coordinate frame, connected with the geomagnetic field $\boldsymbol{H}_{0}$, are presented in Fig. 3 (daytime, normal solar activity). In the simulations, the value of the electron cyclotron frequency has been taken as $\omega_{H e}=6 \cdot 10^{6} \mathrm{~s}^{-1}\left(H_{0}=0.35 \mathrm{Oe}\right)$. Note that the minimal vertical scale of change in the components of the dielectric permittivity tensor is $1 \mathrm{~km}$. 


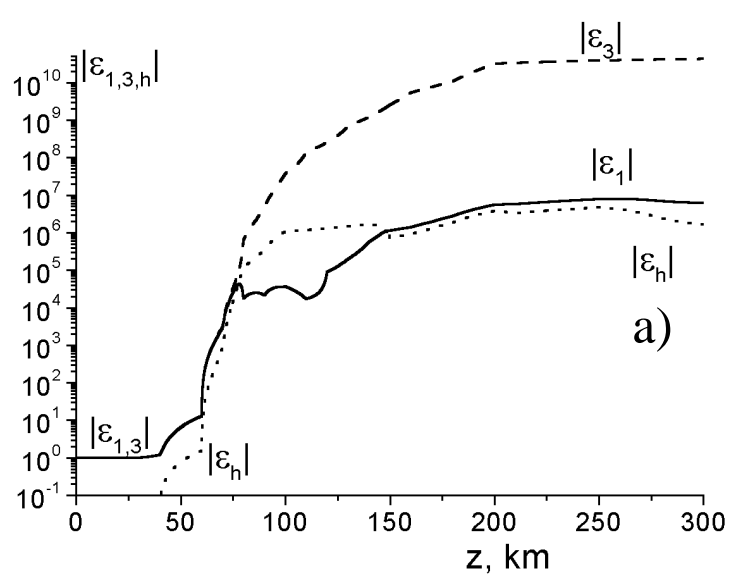

b)

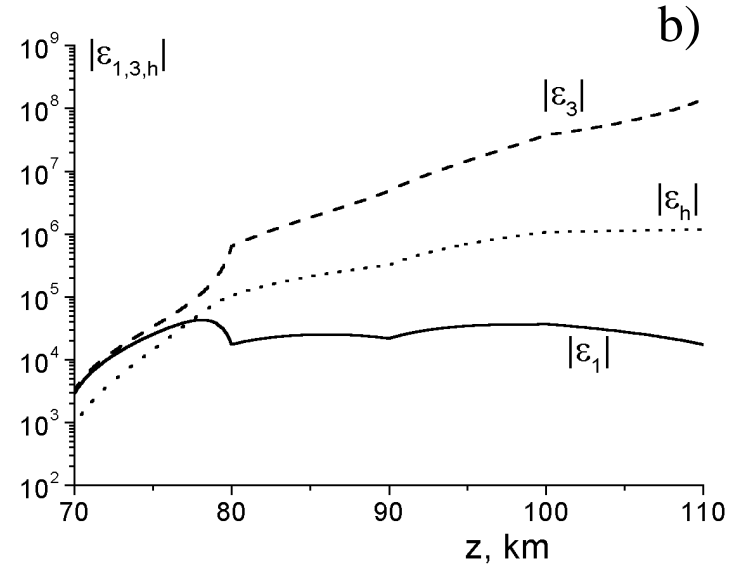

Fig. 3. Vertical dependencies of the absolute values of components of complex tensor of dielectric permittivity of atmosphere and ionosphere at $\omega=50 \mathrm{~s}^{-1}$ used in the simulations (daytime, normal solar activity) (a). The detailed dependence for the heights $70 \div 110 \mathrm{~km}$ (b).

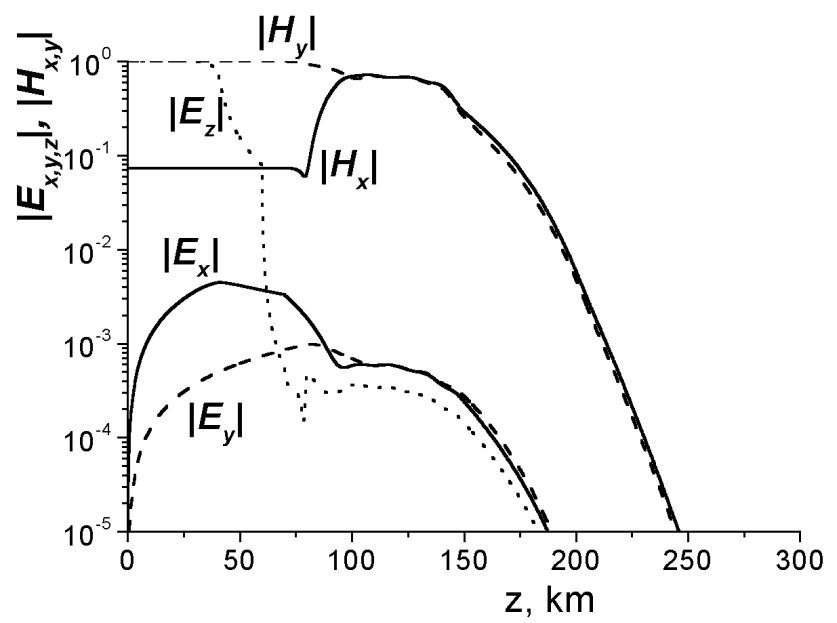

Fig. 4. The dependencies of components $\left|E_{x, y, z}\right|$ and $\left|H_{x, y}\right|$ on the vertical coordinate $z$ under the inclination of the geomagnetic field $\Theta=30^{\circ}$ (daytime, normal solar activity).

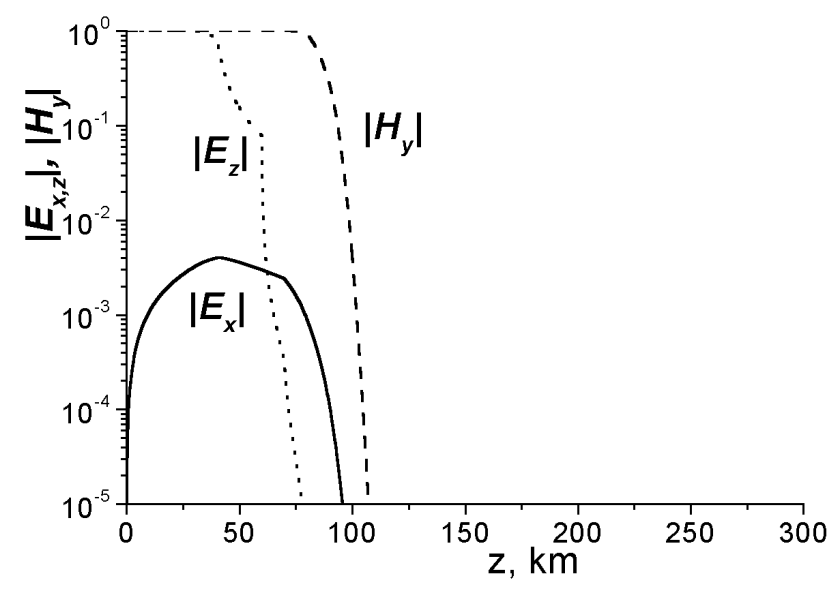

Fig. 5. A dependence of components $\left|E_{x, y, z}\right|$ and $\left|H_{x, y}\right|$ on the vertical coordinate $\boldsymbol{z}$ in the absence of geomagnetic field $\boldsymbol{H}_{0}=0$ (daytime, normal solar activity).

Vertical distributions of electric and magnetic field components of the mode have been calculated under various orientations of the geomagnetic field $H_{0}$, with respect to the vertical axis $O Z$ (angle $\Theta)$ and of the plane $\left(O Z, H_{0}\right)$ with respect to the $O X$ axis (angle $\Phi$ ), see Fig. 1. A penetration of the magnetic components $H_{x, y}$ into the ionosphere depends essentially on the inclination angle $\Theta$. A dependence of the penetration of the magnetic components on the angle $\Phi$ is unessential. In Fig. 4, dependencies of $\left|E_{x, y, z}\right|,\left|H_{x, y}\right|$ on the vertical coordinate $z$ are given for the value of the inclination angle $\Theta=30^{\circ}$ and for $\Phi=30^{\circ}$. The normal daytime conditions are assumed here (Rich and Basu, 1985). The vertical magnetic component $H_{z}$ is quite small: $\left|H_{z}\right| \approx\left|E_{y}\right|$.

At the Earth's surface the components $E_{z}$ and $H_{y}$ possess the greatest values, as well-known earlier (Bliokh et al., 1980; Nickolaenko and Hayakawa, 2002). But the vertical distributions of the electric and magnetic components differ from each other. Moreover, the presence of the geomagnetic field changes essentially the vertical distributions of the horizontal magnetic field components, as shown in Fig. 5, where the vertical distributions of the field components are given in the absence of the geomagnetic field, to compare with Fig. 4. The data of Fig. 5 coincide with the results of the survey (Sentman, 1995) and the papers cited there. Independent of the presence or absence of the geomagnetic field, at the heights $z>50 \mathrm{~km}, E_{z}$ decreases quickly and it becomes of the same order as the $E_{x}$ and $E_{y}$ components. The electric field of the mode does not penetrate highly into the ionosphere, as was known earlier (Sentman, 1995). Moreover, the dependence of $E_{z}(z)$ coincides with the dependence obtained in the paper by Sentman (1990), where a two-scale exponential approximation of isotropic conductivity of the ionosphere was used. But the magnetic field components $H_{x, y}$ can penetrate into the E-layer of the ionosphere up to the heights $\mathrm{z} \approx 150 \mathrm{~km}$, (compare Figs. 4 and 5). Under the oblique direction of the geomagnetic field, a penetration of magnetic components becomes weaker, but it also exceeds 

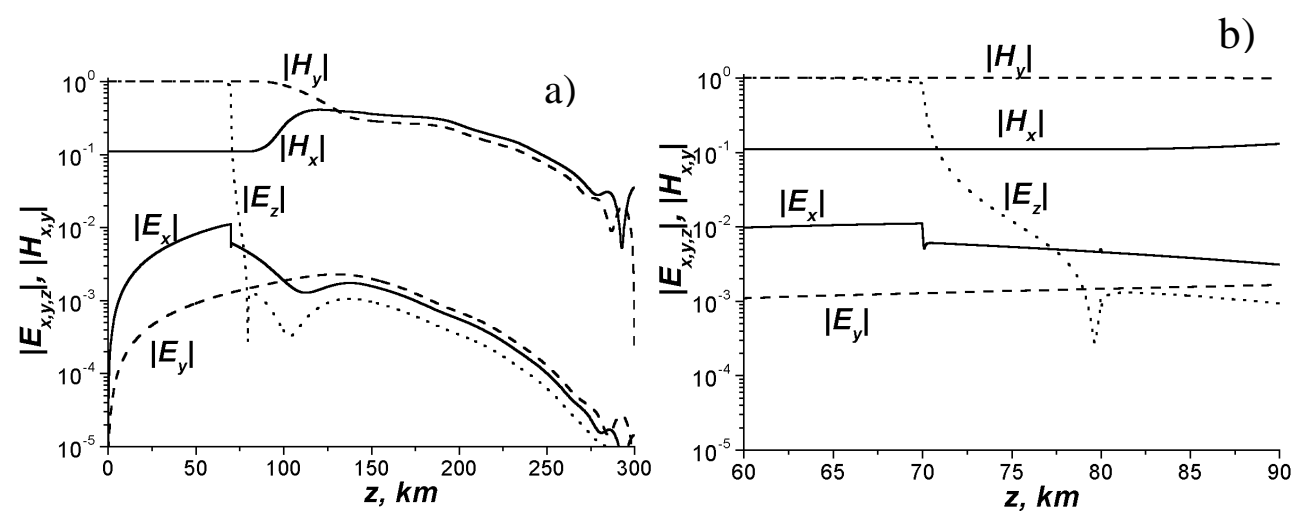

Fig. 6. A dependence of components $\left|E_{x, y, z}\right|$ and $\left|H_{x, y}\right|$ on the vertical coordinate $z$ under $\Theta=30 \circ$ (nighttime) (a). The detailed dependence for the heights $60 \div 90 \mathrm{~km}$ (b).
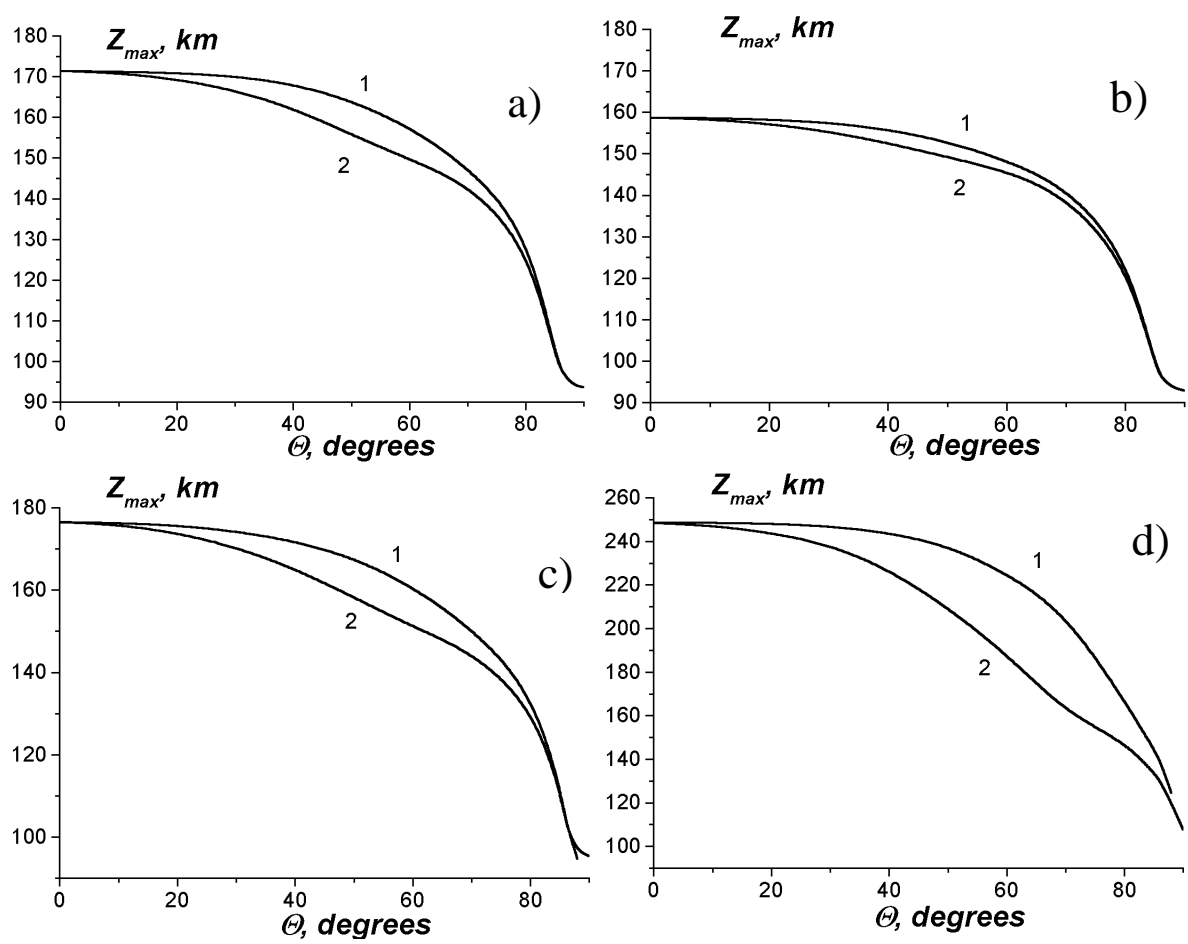

Fig. 7. Dependencies of the penetration heights of the components $H_{x, y}$ of the Schumann resonance on the inclination angle $\Theta$ of the geomagnetic field. Part (a) is for the daytime (normal solar activity); (b) is for daytime (maximum solar activity); (c) is for the daytime (minimum solar activity); (d) is for the nighttime. Curve 1 is the penetration height for $H_{x}$ component; curve 2 is the penetration height for $H_{y}$. For the daytime, the penetration height for the $E_{z}$ component is $56 \mathrm{~km}$. For the nighttime, it is $71 \mathrm{~km}$.

the values obtained in the absence of the geomagnetic field. At the heights $z>50 \mathrm{~km}$ the components $E_{x, y}$ are of the same order as $E_{z}$, also $H_{x}$ is of the same order as $H_{y}$. Therefore, perturbations of the conductivity of the ionosphere D-layer may change the vertical profiles of both electric and magnetic components of the Schumann resonances; the variations of the conductivity of the ionosphere E-layer may lead to the changes in the vertical profiles of magnetic components.
In Fig. 6, the vertical distributions of the field components are presented at the nighttime (normal solar activity). The inclination angle is also $\Theta=30^{\circ}$. The use of more smooth profiles of the electron concentration $n(z)$ at the heights $60 \div 110 \mathrm{~km}$ does not change the vertical dependencies of the magnetic components $H_{x}, H_{y}$ but only leads to smoother dependencies of $E_{x}$ and $E_{y}$ components of the electric field. 
In Fig. 7 the dependencies of the penetration heights on the inclination angle $\Theta$ are given for the normal solar activity, maximal and minimum solar activity (daytime), and the normal solar activity (nighttime). The penetration height is estimated as the value of the vertical coordinate $z$, where $\left|H_{x}\right|$ or $\left|H_{y}\right|$ is $0.1\left|H_{y}(z=0)\right|$. One can see some dependence of a penetration height of horizontal magnetic field components on the variations of the profiles of the electron concentration at the daytime (Figs. 7a,b,c). An essential difference is between the dependencies of the horizontal magnetic field components on the coordinate $z$ at the daytime and the nighttime. The last fact may be explained by the absence of the ionosphere D-layer at the nighttime (Rich and Basu, 1985; Al'pert, 1972). Thus, the horizontal magnetic components of the Schumann resonance possess, at the same time, different penetration heights at different places on the Earth. For the daytime, the penetration height for $E_{z}$ component is $55 \mathrm{~km}$, independent of the solar activity; for the nighttime, it is $70 \mathrm{~km}$.

The maximal penetration takes place at $\Theta \approx 0^{\circ}$, namely, under a vertical direction of the geomagnetic field. It is interesting that at the nighttime, the horizontal magnetic components penetrate deeply into the F-layer (see Figs. 6 and Fig. 7d) This fact may lead to additional dissipation of the Schumann resonances.

To estimate the influence of the curvature of the Earth's surface on the penetration of the magnetic field components into the ionosphere, the spherical geometry has also been investigated. A simple model situation has been considered when the geomagnetic field is directed vertically upwards and is constant. The results of the simulations have confirmed the possibility of the penetration of horizontal components of the magnetic field up to the heights of $120 \div 200 \mathrm{~km}$.

\section{Conclusions}

The simulations of the penetration of the electric and magnetic components of the first Schumann resonance mode into the ionosphere in the cavity Earth - ionosphere have been made in the case of possible daytime and nighttime variations of the conductivity in the ionosphere D- and E-layers. The magnetic components can penetrate into the E-layer of the ionosphere up to the heights $z=150 \div 240 \mathrm{~km}$, with a dependence on the local orientation of the geomagnetic field with respect to the vertical axis. Therefore, variations of the concentration of electrons in the D-layer $(z \sim 60 \mathrm{~km})$ may change the profiles of electric components of the global Schumann resonances; perturbations of the E-layer $(z \sim 100 \div 150 \mathrm{~km})$, due to magnetosphere-ionosphere coupling, may change the profiles of the magnetic components of the global Schumann resonances.

The total energy of the EM resonant mode includes both electric and magnetic parts. Because the magnetic field of the Schumann resonance penetrates up to the heights $150 \div 240 \mathrm{~km}$ in the presence of the geomagnetic field, it is necessary to take into account this fact when calculating the excitation of the resonant mode by thunderstorm activity. The strong anisotropy of the effective dielectric permittivity at the heights $z \geq 70 \mathrm{~km}$ is also important for estimations of quality factors of Schumann resonance modes and for determining the regions of maximal dissipation of ELF electromagnetic waves under the propagation in the Earthionosphere cavity.

Acknowledgements. Topical Editor M. Lester thanks two referees for their help in evaluating this paper.

\section{References}

Al'pert, I. L.: Radio Wave Propagation and the Ionosphere. Nauka, Moscow, 1972.

Bliokh, P. V., Nickolaenko, A. P., and Filippov, Yu. F.: Schumann Resonances in the Earth-Ionosheric Cavity. Peter Peregrinus, London, 1980.

Füllekrug, M. and Constable, S.: Global triangulation of intense lightning discharges, Geophys. Res. Lett., 27, 333-336, 2000.

Füllekrug, M. and Reising, S. C.: Excitation of Earth-Ionosphere cavity resonances by sprite-associated lightning flashes, Geophys. Res. Lett., 25, 4145-4148, 1998.

Hayakawa, M.: Atmospheric and Ionospheric EM Phenomena. TerraPub, Tokyo, 1999.

Madden, T. and Thompson, W.: Low frequency electromagnetic oscillations of the Earth-ionosphere cavity, Reviews of Geophysics, 3, 211-256, 1965.

Mushtak, V. C. and Williams, E. R.: ELF propagation parameters for uniform models of the Earth - Ionosphere waveguide, J. Atmos. S.-P., 64, 1989-2001, 2002.

Nickolaenko, A. P.: Modern aspects of Schumann resonance studies, J. Atmos. S.-P., 59, 805-817, 1997.

Nickolaenko, A. P. and Hayakawa, M.: Resonances in the Earth Ionosphere Cavity. Kluwer, Dordrecht, 2002.

Rich, F. J. and Basu, Su.: Ionospheric Physics, Handbook of Geophysics and Space Environment, Chapter 9. Air Force Geophysics Lab., US Air Force, 1985.

Roldugin, V. C., Maltsev, Y. P., Vasiljev, A. N., et al.: Changes of Schumann resonance parameters during the solar proton event of 14 July 2000: J. of Geophys. Res., A108(1103), doi:1029/2002JA009495, 2003.

Sentman, D. D.: Schumann resonance effects of electrical conductivity perturbations in an exponential atmospheric/ionospheric profile, J. Atmospheric and Terrestrial Phys., 45, 1, 55-65, 1983.

Sentman, D. D.: Approximate Schumann resonance parameters for a two-scale-height ionosphere, J. Atmospheric and Terrestrial Phys., 52, 1, 35-43, 1990.

Sentman, D. D. and Fraser, B. J.: Simultaneous observations of Schumann resonances in California and Australia: evidence for intensity modulation by the local height of the D region, J. Geophys. Res., A96(9), 15973-15 982, 1991.

Sentman, D. D.: Schumann Resonances, Handbook of Atmospheric Electrodynamics, 1, CRC Press, Boca Raton, CA, 1995.

Vainshtein, L. A.: Electromagnetic Waves. Moscow, Radio i Svyaz, 1988. 